\title{
HISTOIRE DE LA TÉLÉMÉTRIE LASER TERRE-LUNE
}

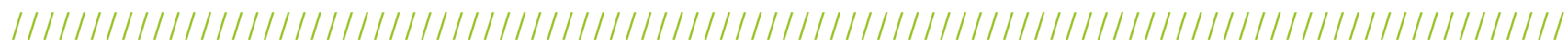

\author{
Julien CHABE ${ }^{\mathbf{1 *}}$, Adrien BOURGOIN ${ }^{2}$, et Nicolas RAMBAUX ${ }^{3}$ \\ ${ }^{1}$ Université de la Côte d'Azur, Observatoire de la Côte d'Azur, CNRS, IRD, Géoazur, Caussols, France \\ ${ }^{2}$ University of Bologna, Dipartimento di Ingegneria Industriale, Forli, Italy \\ ${ }^{3}$ IMCCE - Observatoire de Paris, PSL Université, Sorbonne Université, CNRS, Paris, France \\ * chabe@geoazur.unice.fr
}

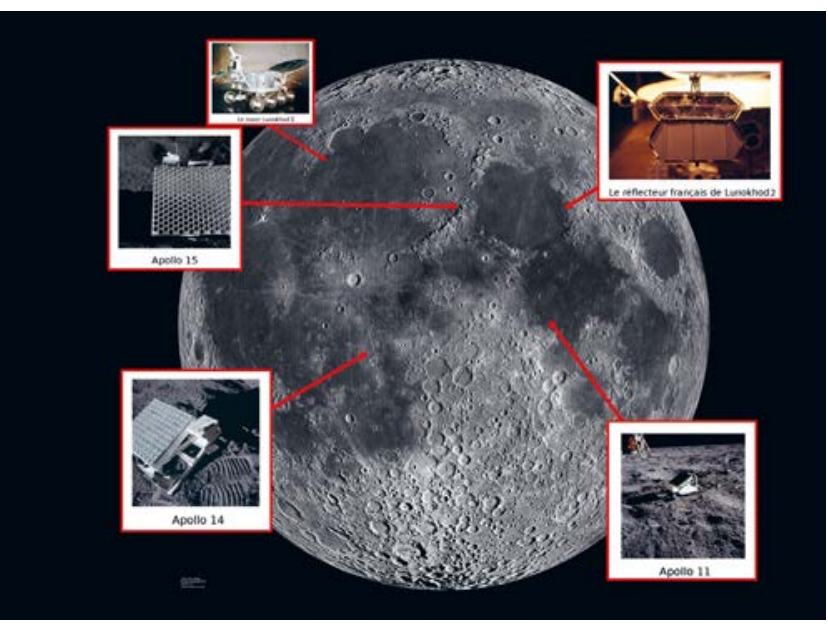

Fin des années 1950, sous l'initiative de R.H. Dicke, la mesure télémétrique Terre-Lune fut initialement pensée pour tester la théorie de la Relativité Générale (RG) au travers de la variation de la constante gravitationnelle et du principe de Mach. Plus tard, K. Nordtvedt démontra qu'une telle expérience permettrait également de tester profondément la RG à travers un effet qui porte aujourd'hui son nom : l'effet Nordtvedt. Au fur et à mesure des développements technologiques, la télémétrie laser a permis de tester bien d'autres aspects fondamentaux de la théorie. En outre, elle a permis d'en apprendre davantage sur la physique du système Terre-Lune et constitue aujourd'hui un outil géophysique à part entière.

https://doi.org/10.1051/photon/202010326

Article publié en accès libre sous les conditions définies par la licence Creative Commons Attribution License CC-BY (https://creativecommons.org/licenses/by/4.0), qui autorise sans restrictions l'utilisation, la diffusion, et la reproduction sur quelque support que ce soit, sous réserve de citation correcte de la publication originale.

\section{HISTORIQUE DES DÉVELOPPEMENTS EXPÉRIMENTAUX}

Des expériences ont eu lieu dès 1962 au Massachusetts Institute of Technology (L.D Smullin et G. Fiocco) pour mesurer la distance Terre-Lune par le temps de vol aller-retour d'une impulsion laser réfléchie directement par la surface lunaire. En 1965, lobservatoire de Crimée en URSS parviendra de cette façon, à une précision de 200 m sur la mesure, limitée par la topographie approximative de la Lune. La télémétrie laser lunaire (Lunar Laser Ranging : LLR) commence réellement lorsque J. Faller, un étudiant de Dicke à l'Université de Princeton, propose l'instrumentation à base de « coins de cube ».
Le premier panneau a été déployé par Buzz Aldrin le 21 juillet 1969. Le $1^{\text {er }}$ août 1969, l'observatoire de Lick obtient les premiers échos laser depuis le site d'Apollo 11. L'observatoire de McDonald obtient des résultats similaires le 20 août 1969 [1]. Dans la nuit du $1^{\text {er }}$ novembre 1969, une équipe française enregistra des échos lasers depuis le réflecteur d'Apollo 11 à l'Observatoire du Pic du Midi.

En parallèle, le CNES commence une collaboration avec l'URSS afin de développer les rétroréflecteurs destinés à équiper les rovers soviétiques Lunokhod. Réalisé par l'entreprise Aérospatiale à Cannes, le réflecteur du rover Lunokhod 1, renvoie ses premiers échos laser vers l'Observatoire de Crimée le 5 décembre 1970. Par la suite, la surface lunaire sera équipée en nouveaux réflecteurs avec les missions Apollo 14, Apollo 15 en 1971 et Luna 21 (Lunokhod 2) en 1973. Le programme Apollo prévoit un observatoire à Hawaï (Haleakala) dédié à la LLR, mais les retards s'accumulent et les premières mesures sont donc réalisées en intégrant des systèmes lasers et des appareils de détection à des télescopes d'observation astronomique existants (de diamètre supérieur à 1,5 m). L'observatoire de McDonald se chargera des mesures de routine jusqu'en 1985. La précision évaluée à l'époque est de $15 \mathrm{~cm}$ sur la distance Terre-Lune. Cependant, pour exploiter au maximum le potentiel 
scientifique de cette mesure, la communauté scientifique prend conscience qu'un réseau d'instruments dédiés à cette activité doit être développé sur le long terme. En 1983, la première station dédiée à la LLR « McDonald Laser Ranging Station » (MLRS1) est opérationnelle. En France, le centre d'Etudes Géodynamiques et Astronomiques (CERGA) est inauguré en 1974 à Grasse et les instruments sont installés sur le plateau de Calern à $20 \mathrm{~km}$ environ de la ville de Grasse (Fig. 1). La station laser dédiée à la LLR y produira sa première mesure de distance en 1981.

De 1962 au milieu des années 1980, le laser à rubis est l'outil qui permet des impulsions courtes et d'énergie suffisamment élevée pour atteindre lorbite lunaire (voir encart « Un défi technique »). Les premières expériences sur la surface lunaire aux USA sont d'ailleurs réalisées avec les premiers modèles de la société Korad développés par Théodore Maiman, pionnier dans le développement des lasers, et équipés d'un système Q-switch à liquide. Bien que le laser Nd-YAG (1964) délivre un nombre de photons par impulsion plus important, l'efficacité des photomultiplicateurs utilisés à $1,06 \mu \mathrm{m}$ est 100 fois plus faible quà $700 \mathrm{~nm}$. Le processus de génération de second harmonique (1961) à cette longueur d'onde est déjà envisagé mais il est d'abord jugé trop complexe à mettre en œuvre. La station d'Haleakala est la première en 1984, à produire des données avec un laser Nd-YAG doublé (impulsions de 200 ps), leur précision est inférieure à $10 \mathrm{~cm}$ (Fig. 2). Cette technologie est alors adoptée par l'ensemble des stations avant la fin des années 80 . Le reste des progrès est attribuable aux améliorations sur l'ensemble des sous-systèmes qui constituent une « station laser Lune » (horloge, détecteur et électronique de détection, progrès sur les rattachements topographiques et la compensation des biais locaux). Depuis 2015, la station française emploie la longueur d'onde native du Nd-Yag via des détecteurs simple photon ayant un bruit temporel compatible avec la précision nécessaire. L'intérêt de cette longueur d'onde n'est pas dans l'amélioration de la précision mais dans le rapport signal à bruit. L'infrarouge permet d'améliorer léchantillonnage du cycle lunaire, là où le vert permettait difficilement d'obtenir des données en dehors des quartiers des phases de la Lune. L'infrarouge permet aussi d'échantillonner de manière homogène sur les 5 réflecteurs lunaires, un point important pour la mesure du mouvement de rotation de la Lune.

\section{PHYSIQUE FONDAMENTALE}

La RG se base sur deux grands principes. Le premier est le principe d'équivalence d'Einstein qui permet d'assimiler la gravitation à la métrique de l'espace-temps. Le second est lié à l'unicité de la métrique dont la dynamique est décrite par les équations d'Einstein. Parmi l'ensemble des théories de la gravitation qu'il est possible de construire, seule la RG semble être en mesure de satisfaire simultanément ces deux aspects ; fait que l'on qualifie de version forte du principe d'équivalence. Les grandes théories d'unification, qui visent à formuler une théorie quantique

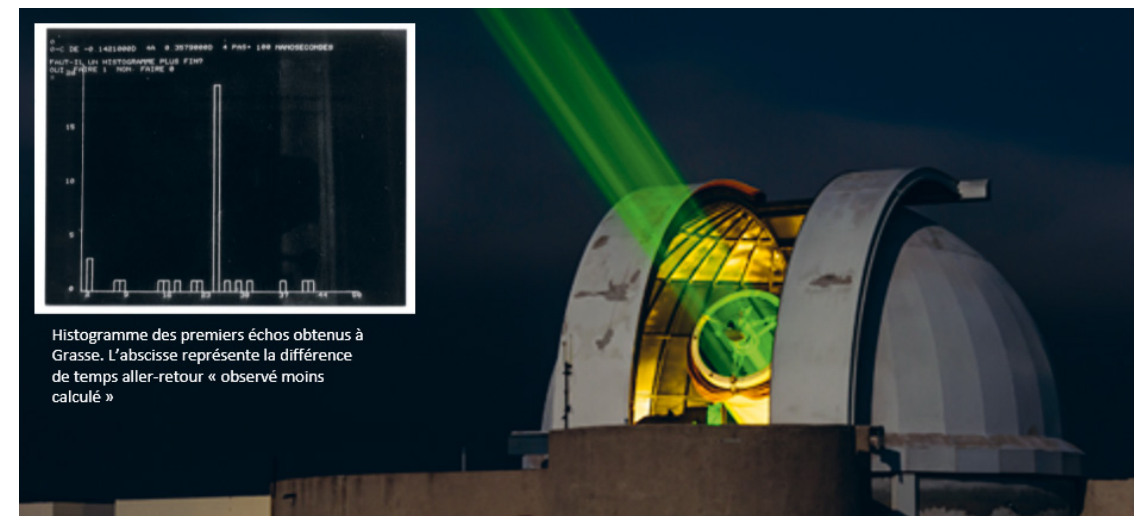

Figure 1. Station française (Grasse) en train de réaliser une mesure télémétrique des rétroréflecteurs lunaires (crédit photo: Cyril Fresillon/ Geoazur / FirstTF/ CNRS Photothèque). 


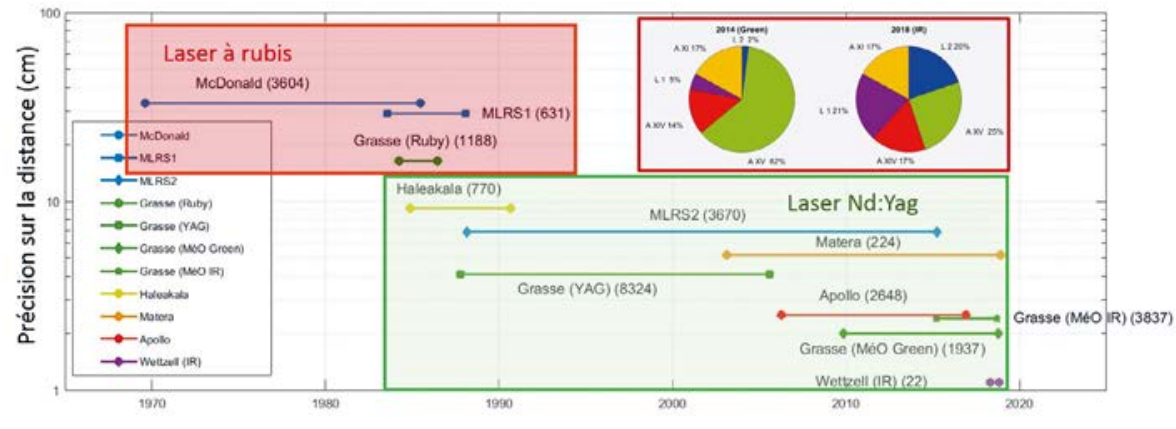

de la gravitation, semblent s'accorder sur le fait qu'à plus ou moins haut niveau d'énergie, l'ensemble de ces principes devrait être violé. La précision des données LLR permet alors de contraindre ces violations.

En 1961, motivés par le principe de Mach, Brans et Dicke formulèrent une théorie alternative de la gravitation satisfaisant le principe d'équivalence, mais dans laquelle la constante de gravitation (G) est désormais une entité dynamique dont la valeur est déterminée par le champ scalaire additionnel. Les données LLR ont cependant montré que la variation relative de $\mathrm{G}$ était nulle avec une précision de l'ordre de $10^{-13}$ [2], comme le prévoit la RG.

Le principe d'équivalence s'appuie, entre autres, sur le principe d'équivalence faible qui stipule que la trajectoire suivie par un corps d'épreuve en chute libre est indépendante de sa structure interne ou de sa composition. En théorie Newtonienne, cet énoncé est équivalent à l'égalité entre la masse inertielle $\left(\mathrm{m}_{\mathrm{i}}\right)$ et la masse gravitationnelle $\left(\mathrm{m}_{\mathrm{g}}\right)$. La LLR a permis de vérifier la validité de cette égalité à hauteur de $10^{-13}$ [3] ; grâce à la mission MICROSCOPE, l'égalité a même pu être vérifiée à hauteur de $10^{-14}$.

En 1968, K. Nordtvedt proposa de tester la version forte du principe d'équivalence. Il démontra que la LLR permettrait de mesurer le ratio $\mathrm{m}_{\mathrm{g}} / \mathrm{m}_{\mathrm{i}}$ de la Terre avec une précision telle, qu'il serait alors possible de détecter la contribution de lénergie gravitationnelle propre de la Terre. Si une telle énergie contribuait en proportion différente entre $\mathrm{m}_{\mathrm{g}}$ et $\mathrm{m}_{\mathrm{i}}$, lorbite lunaire nous apparaîtrait polarisée dans la
Figure 2.

Évolution de la précision des mesures de distance Terre-Lune pour chaque station par rapport à une éphéméride lunaire avec entre parenthèse le nombre de mesures. En haut à droite, comparaison de la répartition des mesures sur les 5 réflecteurs entre le vert (532 $\mathrm{nm}$ ) et l'infrarouge (1064 nm) pour la station de Grasse.

\section{UN DÉFI TECHNIQUE}

direction du Soleil. Cependant, parce que les compositions internes de la Terre et de la Lune sont différentes, la mesure de $\mathrm{m}_{\mathrm{g}} / \mathrm{m}_{\mathrm{i}}$ ne suffit pas. Les résultats d'une expérience de balance de torsion ont du être combinés avec les analyses télémétriques LLR afin de s'assurer que l'énergie gravitationnelle propre de la Terre contribuait bel et bien en proportion identique entre $\mathrm{m}_{\mathrm{g}}$ et $\mathrm{m}_{\mathrm{i}}$ jusquà une précision de $10^{-4}$ [2].

La RG ainsi que le modèle standard des particules s'appuient sur une symétrie fondamentale de la relativité restreinte : la symétrie de Lorentz. En RG cette propriété apparaît localement aussi bien au niveau du principe d'équivalence que dans les équations d'Einstein. Cependant, là encore, les grandes théories d'unification prédisent une violation de cette

La LLR se base sur la mesure précise du temps de vol aller-retour d'une impulsion laser émise depuis un télescope vers un rétroréflecteur catadioptrique. Pour avoir une chance de détecter un signal, les impulsions laser utilisées sont relativement énergétiques $(1 \mathrm{~J})$ mais la mesure reste difficile à mettre en œuvre. Le faisceau laser émis a une divergence minimale d'une à trois secondes d'arc en fonction des conditions atmosphériques (seeing). Lorsqu'il atteint la surface lunaire, la tache laser est une figure de tavelure de $6 \mathrm{~km}$ de diamètre, si bien qu'en moyenne seule une fraction $\left(10^{-9}\right)$ de la lumière est réfléchie par le réflecteur. Sur le chemin retour, la divergence est supérieure à 10 secondes d'arc. Un télescope $\mathrm{d}$ ' $1,5 \mathrm{~m}$ de diamètre collecte alors seulement $10^{-9}$ de la lumière renvoyée depuis la Lune. En pratique, les conditions atmosphériques variables (transparence, turbulence atmosphérique) imposent un bilan de liaison réel entre $10^{-19}$ et $10^{-20}$. De telles pertes nécessitent de filtrer drastiquement la lumière parasite. Les photons reçus passent par trois types de filtres : un premier filtre de champ de l'ordre de dix secondes d'arc, un second filtre de type interférentiel $(0,1 \mathrm{~nm})$ centré sur la longueur d'onde du laser et un filtre temporel qui déclenche les photodiodes à avalanche (en mode Geiger) dans une porte de $100 \mathrm{~ns}$ autour de l'instant estimé du retour des photons. Enfin, pour atteindre une exactitude de l'ordre du centimètre sur une distance moyenne de $384000 \mathrm{~km}$, la chronométrie doit être basée sur une référence de fréquence ultrastable, avec une stabilité relative meilleure que $10^{-12}$ comme celle délivrée par un maser à hydrogène. D'autres facteurs vont affecter la précision, l'exactitude et la stabilité de la mesure. L'incertitude liée à la méconnaissance de l'indice de réfraction de l'atmosphère sur le trajet lumineux peut générer une erreur de plusieurs millimètres, de même pour le bruit temporel des détecteurs. La croisée des axes du télescope (le seul point fixe au sol pendant un suivi de la Lune et donc pris pour référence) doit être connu avec une incertitude inférieure au centimètre dans le référentiel terrestre. 
symétrie fondamentale. Récemment, l'analyse des données a permis de vérifier que la dynamique orbitale de la Lune satisfait bien à la symétrie de Lorentz dans les deux cas jusquà des précisions allant de $10^{-8}$ à $10^{-12}$ [4].

\section{CONTRIBUTIONS GÉOPHYSIQUE ET SÉLÉNOPHYSIQUE}

Bien qu'initialement dédiée aux tests de physique fondamentale, la LLR a permis de nombreuses retombées scientifiques en géophysique. Lajustement de modèle dynamique du système Terre-Lune (orbite et rotation) aux données LLR permet de construire les éphémérides lunaires tel que l'éphéméride INPOP, Intégration Numérique Planétaire de l'Observatoire de Paris [5]. La précision des éphémérides a été augmentée de plusieurs ordres de grandeur grâce aux données LLR et à la continuité des mesures. Cette précision a permis, entre autres, de déterminer léloignement de la Lune à $3,81 \pm 0.02 \mathrm{~cm} / \mathrm{an}$ dû essentiellement aux marées terrestres. Les premiers tirs lasers ont également contribué à une meilleure compréhension de la rotation de la Terre en identifiant une oscillation de 50 jours liée à l'échange de moment cinétique entre la Terre solide et l'atmosphère qui a stimulé les recherches dans ce domaine.

En science lunaire (ou sélénophysique), la LLR a également joué un rôle majeur dans le renouvellement de notre vision de la structure interne de notre satellite. La Lune est en rotation synchrone autour de la Terre et sa rotation peut se décrire en termes d'oscillations périodiques baptisées librations. L'amplitude de ces librations dépend principalement de la répartition de masse à l'intérieur de la Lune et fournissent, en combinant avec les mesures de gravimétrie spatiale, des informations sur la variation de densité dans la Lune. De plus, la grande sensibilité des données LLR permet de quantifier la dissipation dans notre satellite [6]. Dans les années 1980, il a été démontré que cette dissipation avait pour origine les marées solides et la friction à l'interface d'un manteau solide et d'un noyau fluide. C'était là une première signature indirecte de la présence d'un noyau fluide dans la Lune. La présence du noyau fluide a été confirmée en 2011 par deux études indépendantes basées sur la ré-analyse des données des sismomètres des missions Apollo. Cependant, les modèles de noyau sont très différents et laccumulation des données LLR depuis plus de 50 ans a permis en 2019 de trancher en faveur d'un noyau liquide de $381 \pm 12 \mathrm{~km}$ de rayon avec une précision inégalée par les autres méthodes géophysiques appliquées à la Lune [7].

\section{CONCLUSION}

En 1973, Neil Armstrong écrivait déjà de la LLR: «L'expérience était naturellement simple dans son concept et remarquablement complexe dans son exécution. La diversité des problèmes auxquels s'appliquent les informations issues de cette expérience dépasse l'imagination. ». Les développements réalisés et les résultats acquis en physique fondamentale et du système Terre-Lune depuis n'ont fait que confirmer cette observation.

\section{RÉFÉRENCES}

[1] J. Faller, I. Winer, W. Carrion et al., Science 166, 99 (1969)

[2] S.M. Merkowitz, Living Rev. Relativ. 13, 7 (2010)

[3] V. Viswanathan, A. Fienga, O. Minazzoli et al., Mon. Not. R. Astron. Soc. 476, 1877 (2018)

[4] A. Bourgoin, A. Hees, S. Bouquillon et al., Phys. Rev. Lett. 117, 241301 (2016) ;

A. Bourgoin, C. Le Poncin-Lafitte, A. Hees et al., Phys. Rev. Lett. 119, 201102 (2017)

[5] A. Fienga, P. Deram, V. Viswanathan et al., Notes Scientifiques et Techniques

de l'Institut de Mecanique Celeste 109 (2019)

[6] J.G. Williams, D.H. Boggs, C.F. Yoder et al., J. Geophys. Res. 106, 27933 (2001)

[7] V. Viswanathan, N. Rambaux, A. Fienga et al., Geophys. Res. Lett. 46, 7295 (2019)

Imegine Optie

Wavefront sensors and

adaptive optics for

optical metrology,

lasers and microscopy

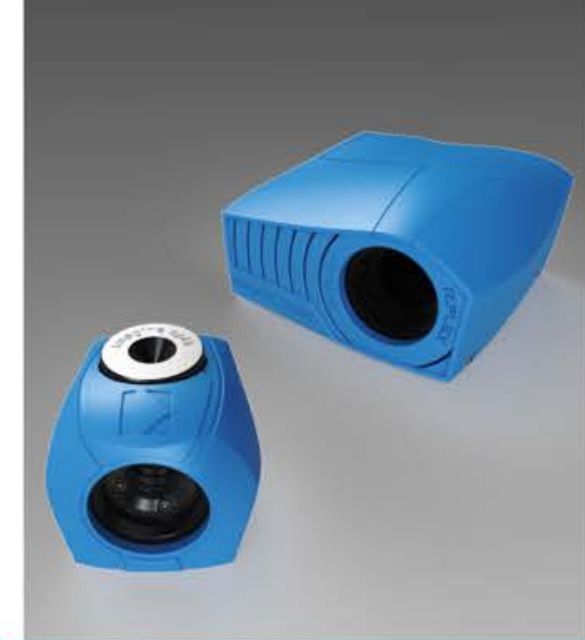

Optical metrology applications
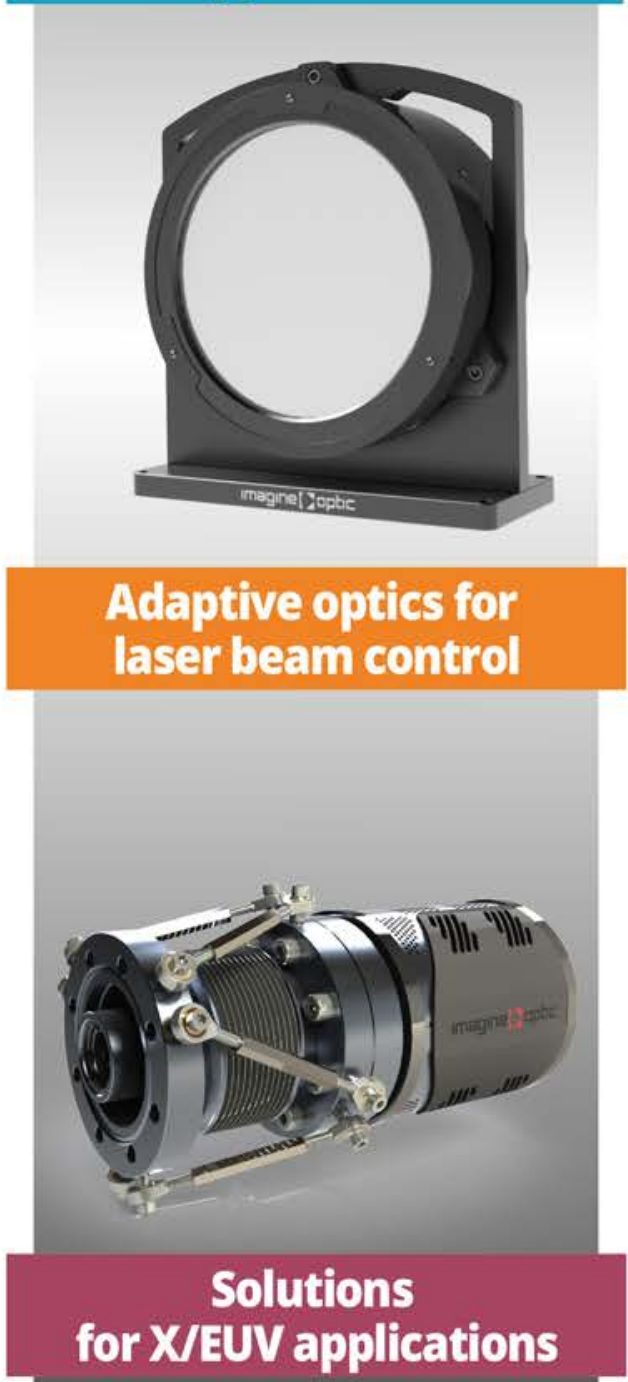

Contact us for more details: contact@imagine-optic.com or +33164861560 